\title{
Critical Care of the Morbidly Obese in Disaster
}

James Geiling, MD, FCCM ${ }^{a, b, *}$

\section{KEYWORDS}

- Morbidly obese • Obese • Disasters • Critical care

- Emergency management $\bullet$ Special populations

The prevalence of obesity in the United States is increasing, with extreme morbid obesity of body mass index (BMI, calculated as the weight in kilograms divided by height in meters squared) greater than 40 increasing twice as fast as obesity in general. With the increased weight comes an increased risk of comorbidities, including type 2 diabetes mellitus, cardiovascular disease, respiratory problems such as obstructive sleep apnea (OSA) or restrictive lung disease, skin disorders such as intertrigo and cellulitis, and urinary incontinence. ${ }^{1}$ Thus, patients exposed to a variety of disasters not only are increasingly overweight but also have an associated number of coexistent medical conditions that require increased support with medical devices and medications.

Recent events support the need to plan for managing these patients. For example, Hurricane lke in 2008 had a high prevalence of obese patients who strained evacuation and shelter systems. Planning for this patient population is now becoming a requirement for relief organizations, with most considering placing them in special-needs shelters. ${ }^{2}$

Although the focus of this discussion is the effect of disasters on the management of the morbidly obese patient, many formerly obese patients may also have an altered response to the stresses of disasters. Many obese patients who fail to respond to

\footnotetext{
The author has no financial conflict of interest to disclose.

Funding: There was no funding for this work.

The opinions and assertions contained herein are those of the author and do not necessarily reflect the views or position of the Department of Veterans Affairs or the academic institutions with which the author is affiliated.

a Veterans Affairs Medical Center, 215 North Main Street, White River Junction, VT 05009, USA

b Dartmouth Medical School, New England Center for Emergency Preparedness, One Medical Center Drive Lebanon, NH 03756, USA

* Dartmouth Medical School, New England Center for Emergency Preparedness, One Medical Center Drive Lebanon, NH 03756.

E-mail address: james.geiling@dartmouth.edu
} 
changes in lifestyle or medications choose to undergo a variety of bariatric surgical procedures. The most common procedures include gastric banding (which may be adjustable), sleeve gastrectomy, Roux-en-Y gastric bypass, and biliopancreatic diversion with duodenal switch. Some patients treated with these procedures suffer from postoperative gastrointestinal complaints, including nausea, vomiting, the dumping syndrome (especially with the Roux-en-Y procedure), and other complaints such as ulcers, bowel obstructions, hernias, and adhesions. ${ }^{3}$ These patients have specific nutritional needs after surgery. Many patients have food intolerances, including intolerance to bread, dairy foods, carbonated beverages, dry or sticky food, or foods of high sugar content. In addition, these patients often require protein-rich foods, and especially in the early postoperative period, they need to avoid dehydration. ${ }^{4}$ Thus, the complicated logistics of nutritional support in a disaster may put these patients at risk for a variety of gastrointestinal complaints, poor wound healing, and dehydration. In addition, besides the routine presentation of these complications at the time of a disaster, the signs and symptoms of these adverse effects may also mimic the signs or symptoms of a variety of common disaster-related complications, including blast abdomen, toxin or chemical ingestion, gastrointestinal complications of biologic agents or acute radiation exposure, or simply the overwhelming effects of the stress of experiencing a disaster. Therefore, especially in these patients, a thorough knowledge of the medical and surgical history becomes important in discerning the effects of the previous surgery from that of the acute event.

\section{HOSPITAL IMPACT}

The scope of the problem may be greater than anticipated. For example, simply recognizing the problem can be especially difficult, because emergency personnel can accurately predict the weight of patients with a BMI greater than 30 only $23 \%$ of the time. ${ }^{5}$ Even internal medicine residents who often treat the medical consequences of obesity do not know the BMI required to diagnose obesity $60 \%$ of the time; $40 \%$ do not even know their own BMI. ${ }^{6}$

Patients presenting to emergency departments during normal daily operations are increasing in number as reflected by national statistics. From 1986 to 2000, patients with a BMI greater than 30 increased from $10 \%$ to $20 \%$ of the population; those with a BMI greater than 40 increased from $0.5 \%$ to $2 \%$. Those with a BMI greater than 50 increased from 1 in 2000 to 1 in 400 persons. More than three-fourths of emergency departments in 2006 reported an increase in the number of these "superobese" patients. These patients require specialized transportation equipment and extra support personnel. Ambulances need special beds, ramps, winches, and other equipments to bring them to the emergency departments. Emergency departments also require expensive equipments such as patient lifters that cost approximately $\$ 18,000{ }^{7}$

\section{DISASTER CRITICAL CARE}

As the patients' weight and mobility requirements increase, so do the resource requirements to meet those needs. Once exposed to a disaster scenario, patients require on-scene treatment and transportation to a facility capable of managing them. Unlike normal events, in which most survivors find their own conveyance to the closest hospital, obese patients will most likely not be able to do that, relying therefore on emergency medical services (EMS). Less critically ill patients may be taken to lower-acuity facilities or, in the setting of a slowly evolving event like pandemic flu, a community surge facility such as an acute care facility. For example, special accommodations may also be needed to get these patients immunized, because it is unlikely 
that they will be able to get to a community vaccine or medication point of distribution (POD). If hospitalization is required, these patients will need to be managed regionally as is done with pediatric or burn patients at present. Regional Medical Control Centers should monitor what facilities can manage these patients and ensure that proper transportation assets are available to assist in their movement. ${ }^{8}$

In addition, community health planners need to be aware of these patients in their community, in the same way they should be aware of patients with home oxygen or medical devices. If these patients need evacuation because of a pending disaster or sudden event, then the local medical control should be proactive in seeking resources to move them to appropriate care or transportation hubs for moving them out of the region. ${ }^{9}$

When disasters occur, the effect on the health care system depends on the nature of the event. In sudden-impact disasters such as transportation accidents, bombings, or fire, large numbers of patients tend to present to the closest facility over a short time span, typically within 3 hours. The hospital is mostly affected in the emergency department and, perhaps, the operating room. However, more protracted events such as pandemic flu would have a more prolonged effect on intensive care units (ICUs). These valuable resources may quickly become overburdened, requiring resources to surge to meet those needs. Ideally, hospitals discharge less critically ill patients to home, long-term care facilities, or even community disaster centers, leaving staffed ICU beds available for those needing this care. However, because those strategies also become overwhelmed, hospitals will need to expand their internal critical care capabilities with creative approaches to surging equipment, staff, and care space, that is, stuff, staff, and space. Because of the increased needs and comorbidities of obese patients, they pose additional challenges to this surge strategy. ${ }^{10}$

\section{Stuff}

Simple measures such as determining vital signs may be challenging in a disaster setting. In a review from France, prehospital management problems of morbidly obese patients included inability to measure blood pressure in $9 \%$, inability to gain venous access in $13 \%$, and difficulty in intubating $20 \%$ of the patients, often requiring advanced airways. ${ }^{11}$ The routine use of noninvasive blood pressure cuffs may not be useful because of the inherent inaccuracies of the cuff's bladder size to arm circumference. $^{12}$ Normal emergency department and ICU procedures may also need additional support, including changed or decreased quality of electrocardiograms, poor radiographic or ultrasonographic imaging, increased reliance on invasive monitoring or procedures, such as diagnostic peritoneal lavage if the patient cannot fit the computed tomographic scanner, and advanced airway support due to oropharynx size or neck mobility limitations. ${ }^{13}$

In 2008, the Task Force on Mass Critical Care reviewed the recommended ventilator characteristics for disaster settings as well as ancillary equipment. ${ }^{14}$ Although review of that information reveals no specific recommendations for the needs of the obese patient, it does point to the requirement to ensure the equipment cached for disaster events do, indeed, fit these patients. The ventilator characteristics should meet such patients' needs, although if noninvasive ventilation is considered (which was not recommended by the Task Force), appropriately sized masks must be available. In addition, monitoring equipments such as blood pressure cuffs and pulse oximeters as well as central venous catheters and other tubes and lines must be of sufficient sensitivity and size to care for these patients.

Pharmacologic caches of emergency medications may not always include those that might be most appropriate for this patient population. For example, opioids for 
pain have a widespread variability in effectiveness with significant interindividual analgesic requirements. Treatment at the higher end of the recommended dosing ranges for a variety of antimicrobials may also be needed. Even corticosteroid use in acute short-term indications may need higher dosing according to adjusted body weight to avoid subtherapeutic effects at lower dosing. ${ }^{15}$ Depending on the number and sizes of these patients in the disaster setting, large quantities of medication may be needed to manage this population. As a result of the increased logistic demands, providing enough medications for all patients could be jeopardized.

Obese patients may be on medications to treat their obesity. These medications include sibutramine, phentermine, diethylpropion, orlistat, bupropion, fluoxetine, sertraline, topiramate, and zonisamide. Side effects of these medications, such as tachycardia, increased blood pressure, insomnia, paresthesia, dyspepsia, diarrhea, flatulence, and abdominal pain may mimic or alter normal findings that result from disaster-related trauma or toxin exposure. ${ }^{16}$ Also, few, if any, of these medications may be in disaster pharmacology caches, resulting in the risk of acute disruption in their availability for these patients.

Beds can be a challenge, especially in shelter or improvised space. Obese patients often require 2 cots that then need devices to keep the patients safe. Because cots are low to the ground, getting the patient on and out of the bed can be difficult for the staff. In addition, these cots tend to lie flat; obese patients often have comorbidities such as congestive heart failure or especially OSA that necessitate an upright position in bed. They have also been noted to have difficulty in clearing secretions when recumbent. ${ }^{17}$ Also, in shelters, observed snoring and OSA in patients have prompted urgent "disaster sleep studies" and have been shown to adversely affect others in the shelter, disturbing their sleep. ${ }^{18}$ Shelters also have limited toilet and bathing facilities. Obese patients may require special accommodations for these routine functions. For example, nurses are often not aware of the weight limitations of toilets or bedside commode, leading to potential patient safety challenges. ${ }^{19}$

The US Department of Health and Human Services has developed and continues to improve several fully equipped Federal Medical Stations (FMS) that can deploy to disaster locations. The FMS provide a 250-bed capability that includes 3 days of medical supplies, equipment, and pharmaceuticals. FMS are deployed to disaster locations and set up in buildings of convenience, such as convention centers, arenas, or warehouses. Although normally configured to manage low-acuity patients, they can also, with additional resources, support more critically ill patients. The FMS that were established in 2008 after the Hurricanes Gustav and Ike had approximately $20 \%$ of their critical patients classified as morbidly obese (Yeskey K, Deputy Assistant Secretary, Director, Office of Preparedness and Emergency Operations, Assistant Secretary for Preparedness and Response, Department of Health and Human Services, personal communication, February 18, 2010), and this diagnosis, which did not exist previously, has been added to electronic health records for patients. As part of the special-needs configuration of FMS, a bariatric set comprising 5 special electric beds and other bariatric items has been prepared. Special equipments in these sets include:

1. Electric beds with $270-k g$ capacity

2. Portable commodes with $450-\mathrm{kg}$ capacity

3. Continuous positive airway pressure (CPAP) machines

4. CPAP masks

5. Obesity gowns

6. Patient hydraulic lifts with $450-\mathrm{kg}$ capacity

7. Transfer bench with $315-\mathrm{kg}$ capacity 
8. Walkers with $315-\mathrm{kg}$ capacity

9. Wheelchairs with $315-\mathrm{kg}$ capacity.

(Donohue J, Emergency Management Specialist/FMS Division Strategic National Stockpile, Office of Public Health Preparedness and Response (OPHPR) Centers for Disease Control and Prevention, personal communication, February 18, 2010).

\section{Staff}

Paramount in managing morbidly obese patients is the safety of the staff. During normal operations, many health care facilities as well as first-responder or transportation assets lack appropriate weight-based assistance devices. Even if available, these patients require increased number of staff to help safely move them. ${ }^{20}$ In disaster settings where medical staff may have limited availability and security, EMS, volunteers, and other nonmedical personnel may be used to assist health care workers in patient movement, lifting, and other physical tasks. ${ }^{17}$

In addition to the increased number of staff required to manage obese patients, the staff themselves are similarly increasing in size along with the general population, putting themselves at risk for injuries and illnesses related to their size. EMS personnel are especially at risk because of the physical demands placed on them, especially in disaster settings. Of almost 400 consecutive fire and ambulance recruits in Massachusetts, all staff had a BMI greater than 25 , with $33 \%$ having a BMI greater than $30 .^{21}$

\section{Space}

The Task Force on Mass Critical Care recommends that in disaster settings, critical care occurs in hospitals and not in field hospitals or contingency facilities in the community because of the special needs of critically ill patients, such as hospital beds, infection-control barriers, specialized equipments (eg, ventilators), oxygen, and other needs. ${ }^{14}$ These needs are especially relevant to the obese patient. However, the repurposing of surge space in a hospital, such as postanesthesia care units, intermediate care units, endoscopy suites, and surgicenters, implies that those spaces have the stuff and staff to manage the patients. As hospitals develop their own mechanisms for managing obese patients, the locations and equipment to manage them, such as patient lifts, may not be universally available in these surge spaces, thereby necessarily accommodating these patients in the few areas already designated for their needs.

\section{Transportation}

Clearly, the morbidly obese patient requires additional or special transportation assets. At home or care facility, large chairs, litters or stretchers, evacuation devices or sleds, or other transport devices must be available. Movement to the ambulance requires special transport stretchers, which need to accommodate a variety of patient positions. Even loading the patient on to the vehicle may require special ramps or winches. ${ }^{22}$ This special equipment is expensive and the additional manpower and time needed to move these patients can be significant. For example, 22 firefighters and EMS personnel reportedly took 2.5 hours to move a woman weighing $347.4 \mathrm{~kg}$ out of the narrow doorway of her town house to the ambulance. ${ }^{7}$

External resources including ground and air vehicles all have specific weight and load restrictions, some of which may further affect movement depending on weather conditions. Bulky and heavier patients put additional strains on vehicles and even require more fuel to carry the increased load. In addition, just because of their size, additional equipments, and extra support persons there is less room for nonobese 
patients; thereby increasing the evacuation needs, such as more vehicles, aircraft sorties, and so on.

Normal evacuation procedures also need to be altered with innovative solutions. A patient weighing $495 \mathrm{~kg}$ required transfer from a rural community hospital to a tertiary care facility for an apparent acute abdomen. Because normal vehicle and transport devices had only $382.5-\mathrm{kg}$ capacity, the community provider solicited the help of the state's national guard. The patient was then transferred in his bed to the large $\mathrm{CH}-47$ Chinook helicopter, the US Army's tandem-rotor heavy-lift helicopter for transport, along with hospital support personnel. ${ }^{23}$ Because of the urgency, a local paramedic unit that was not trained in aeromedical evacuation procedures provided support in the air. On arrival at the receiving facility, the downdraft of the helicopter resulted in receiving personnel being blown about the landing area and local damage to nearby cars (Hinds J, Operations Manager, Dartmouth-Hitchcock Advanced Response Team, personal communication, January 7, 2010). Although the result was a successful patient transfer, the scenario highlights the high resource demands these patients would place on an EMS system when, in a disaster, resources would be severely constrained.

Ideally, emergency medical plans would include vehicles capable of managing these patients. They should be comfortable for the patient, accommodate the increased support persons needed, and ease patient movement without causing lifting injuries. However, the challenge is that such a resource would be expensive to purchase and maintain; expensive to equip, staff, and train; and is in short supply.

Given the requirements and restrictions in moving obese patients, pressureinduced rhabdomyolysis may occur. Although rare, it is a well-described complication of bariatric surgery, and thus the complication could occur not only in the operating room but also under similar circumstances in which patients are immobile for prolonged periods during transport. ${ }^{24}$ Acute compartment syndrome may also develop as a consequence and is difficult to diagnose in already compromised extremities or if patients are sedated and cannot provide adequate history of pain.

\section{MANAGEMENT OF OBESE PATIENTS IN SPECIFIC DISASTERS Trauma, Blast, and Burn Injury}

Traumatic injury is often the consequence of disasters and its effect on the morbidly obese patient must be considered in planning support to these patients. Once hospitalized, severely obese patients with a BMI greater than 30 have higher mortality than obese patients. Increased injury severity score and BMI are independent determinants of outcome with obese patients having increased pulmonary, renal, and thromboembolic complications. ${ }^{25}$ Obese patients who survive also require longer periods of mechanical ventilation and hospitalization. ${ }^{26}$ However, the mortality data on these patients suffering blunt traumatic injury are not consistent. ${ }^{27}$ In fact, although obese patients may have longer hospitalizations, which must be considered in planning health care system capabilities, they do not seem to suffer increased ICU or hospital mortality, which may actually be lower. ${ }^{28}$

Most assume that airway management in trauma patients is compromised by the physical changes and limitations in the morbidly obese. However, data demonstrating this problem are limited. In comparison with lean and overweight patients, a review of airway management experience at a level 1 trauma facility showed no difference in difficult intubations. However, an important caveat is that $92 \%$ of the intubations were conducted by an experienced anesthesia team, with $6 \%$ by experienced emergency department personnel. In short, although classically these patients are 
thought to have challenging airway management issues, in experienced hands this has not been demonstrated. ${ }^{29}$

Blast injuries result in primary (such as tympanic membrane rupture), secondary (from blast projectiles), tertiary (from being projected into an obstacle), and quaternary (from associated injuries such as burns) injuries. Prognostic factors include the magnitude of the explosion, building collapse, time interval to treatment, triage accuracy, and immediacy of medical or surgical support. ${ }^{30}$ Several of these prognostic factors may be affected by the size of the patient, including accuracy of the triage, ability to transport the patient, and the resources needed to get the patient immediately to the operating room. Specific effects of the blast may also be affected by the size of the patient, including the development of intestinal injuries. The potential cushioning effect of a pannus could serve as a protective barrier, yet diagnosis of acute blast abdomen may be challenging depending on ultrasonography or computerized axial tomographic scan capabilities; peritoneal lavage may need to be performed. Large wounds in patients with significant subcutaneous tissue involvement may be predisposed to poor wound healing.

Burn management in these patients should follow normal protocols. But again, the challenges lie in the details. Clearly, with increased body surface area these patients are at risk for more injury and hence higher fluid requirements and infection risk. Obesity itself predisposes these patients to increased morbidity (ie, infection), ventilatory support, insulin requirements, antibiotic usage, and perhaps immunologic markers. ${ }^{31}$ Skin grafting could be potentially problematic, particularly because these patients may already be at risk for decubitus stasis changes. Ideally, these patients would be managed in a burn center, thereby highlighting the transportation challenges discussed earlier.

\section{Natural Disasters and Mass Casualty Crush Syndrome}

Natural disasters have distinct injury patterns associated with the type of disaster. The greatest challenge early in natural disasters is to determine the extent of damage and hence the number of casualties. Initial impressions and estimates, on which health care decisions are made, are often inaccurate. As a result, secondary disasters from misallocation of resources often develop. Clearly, the type of disaster and especially its geopolitical location determine the effect of obese patients on the response effort. For example, in the immediate aftermath of the Haiti earthquake of 2010, there were no obese patients in the native population and hence management of these patients was not an issue. Obesity in the responders, however, did pose challenges, because many of the responsders were not acclimated to the heat or physical demands of the event (Geiling J, personal observation, 2010).

Many natural disasters result in large numbers of crush injuries. Clearly, obese persons are at an increased risk of being exposed because of their baseline-limited mobility. Extraction of these patients poses significant challenges as a result of the increased body surface area being trapped and additional equipment and personnel needed to conduct the extraction. Once trapped, their limbs of increased mass, even if mostly adipose tissue, pose a risk for development of crush injury with the resultant rhabdomyolysis and acute kidney injury. ${ }^{32}$ Compartment syndrome in injured extremities may also develop, especially as patients are extracted and hydrated. The classic findings of pulselessness, pallor, absence of pain, poikilothermia, and paralysis may be difficult to obese extremities, putting additional risk to injured extremities.

\section{Chemical, Biologic, and Radiologic Disasters}

Chemical disasters, not caused by industrial or transportational accidents, result from the use of nerve agents, vesicants, cyanides, pulmonary agents, and riot-control or 
other nonlethal agents. Primary to the management of these patients is to remove them from the contaminated environment and decontaminate them. The challenge of moving these patients as has been discussed in other settings puts them at risk of additional or prolonged exposures. Decontamination can be either dry (mostly accomplished by removing a survivor's clothes) or wet (by having patients washed off with soap and water). Ambulatory patients can do this themselves, but nonambulatory patients require assistance. Again, given their movement constraints, additional support personnel and special equipment must be available to not only decontaminate these special patients but also initiate treatment.

Care for most chemical casualties is supportive airway, breathing with mechanical ventilation if needed, intravenous fluids, and so forth. The challenges in providing this care for obese patients have already been discussed. Several agents have specific antidotes, including atropine and pralidoxime for nerve agents and amyl or sodium nitrite with sodium thiosulfate or hydroxocobalamin for cyanide poisoning. ${ }^{33}$ All of these agents have standard recommended dosing that is not weight based. However, for example, if atropine is recommended to be used in 2-mg intervals until secretions caused by nerve agents decrease, large volumes of medications may be needed to manage these patients.

Biologic disasters result from either the intentional release of a bioweapon or, more likely, a naturally occurring event, such as the outbreak of severe acute respiratory syndrome (SARS) in 2003 or H1N1 in 2009. Management of obese patients in these settings poses no specific change in care other than the challenges faced in the routine outpatient and inpatient or ICU care already discussed. Community response efforts to an outbreak may require isolation or quarantine. Such an intervention by public health authorities would further distance these patients from the care and support they normally require from community resources. Depending on their mobility, they may be unable to attend POD centers that may be established to distribute medications or immunizations, thereby putting them at increased risk of contracting the disease at hand.

Once ill and hospitalized, several classic agents require isolation precautions to minimize spread of the contagion. Smallpox, viral hemorrhagic fevers, and H1N1 or other novel influenzas require airborne and contact precautions, whereas plague and SARS require droplet and contact precautions. Thus, management of the obese patients in these settings requires additional resources not normally needed for normal patients in ICU. For example, the simple act of moving these patients or responding to ventilator alarms or other emergencies requires more personnel, with additional personal protective equipment and supplies. Such gear will already likely be in short supply, thereby already aggravating the shortage. ${ }^{34}$

Radiation disasters can take several forms: a bomb and low levels of radiation that is contaminant purposely implanted to create widespread contamination (through a radiation dispersal device, also known as a dirty bomb), an accidental or intentional release of radiation from a nuclear power plant or processing center, or a nuclear fission device or atomic bomb. In short, bombs or explosions associated with radiation require patient decontamination with removal of clothing and washing the body and acute management of traumatic injuries. The challenges of managing obese patients during decontamination and trauma management have already been discussed.

Unique perhaps to the obese population may be their increased risk of radiation exposure. The basic premise in minimizing radiation exposure is time, distance, and shielding. Thus, injured patients or those with impaired mobility may be unable to move out of a contaminated area, thereby increasing their potential radiation exposure 
and dosage. If associated with trauma, obese patients with impaired wound healing are at increased risk of complications when irradiated because of the adverse interaction between trauma and radiation, that is, increasing radiation exposure worsens outcome in traumatized patients. Once diagnosed with acute radiation syndrome, these patients may require colony-stimulating factors and perhaps even stem cell transplantation. ${ }^{35}$

\section{PALLIATIVE CARE AND MENTAL HEALTH SUPPORT}

Increasingly, disaster settings require additional interventions beside typical medical and surgical support. Clearly, patients with underlying mental health disease, or those subject to the stresses imposed by a disaster, require important mental health support during the acute disaster phase as well as during recovery and reconstitution. The morbidly obese patients have been shown to be at increased risk for depression and hence, in addition to other increased resource needs during disasters, they also need mental health support. ${ }^{36}$ Important also in disaster settings is the provision of pain management and palliative care, a requirement that is not unique to obese patients but nevertheless may pose challenges with additional health care staff attention and pharmacologic needs. ${ }^{37}$

\section{DISASTER TRIAGE AND ALLOCATION OF SCARCE CRITICAL CARE RESOURCES}

Although obese patients require increased support and resources, especially in a disaster setting, with appropriate planning and accommodation, most needs can be achieved through surging capacity of stuff, staff, and space. However, depending on the disaster and resource availability, tough triage decisions may be required when there are simply not enough resources to support all in need.

Given the additional resources, challenges, and scope of the problem in managing morbidly obese patients, under normal daily circumstances, there may be an inherent negative stereotype afforded to these patients. Many nurses believe the patients are not motivated to change their condition, and they do not believe they are effective in helping the patients achieve any behavioral change. Similarly, physicians have adverse opinions especially about the morbidly obese patients. The patients often view the health care community as biased against them, often disrespectful in the care they receive. ${ }^{18}$

Thus, faced with a disaster setting of limited resources and perhaps with some patient care bias in managing obese patients, a transparent process of triaging resources must be developed. Several models have recently been proposed in triaging hospital or ICU resources for nontrauma patients, so-called tertiary triage. Most models focus on use of the sequential organ failure assessment (SOFA) score. Through the use of an intensivist-led multidisciplinary triage team, patients in need of advanced clinical resources are evaluated for inclusion and then exclusion criteria and followed up using the SOFA score to determine illness severity and course. Patients with predicted high mortality or failure to improve, in this model, would be supported with palliative care and have their resources reallocated to other patients with greater chance of survival. Important to note is that none of the models specifically target patient weight as a criterion in determining need or exclusion; what counts is the severity of the illness. ${ }^{38,39}$

\section{SUMMARY}

Management of the morbidly obese patient under normal circumstances requires increased resources such as personnel, supplies, and special equipment. In the often 
resource-constrained setting of disasters, normal support to these patients may be jeopardized. In addition, their underlying condition with limited mobility and comorbidities may increase their likelihood of suffering harm depending on the type and tempo of the disaster. Morbidly obese patients are a special-needs population for whom necessary planning must be done to mitigate the effects of the disaster on them. Prior planning not only addresses appropriate care needs for these patients but also avoids crisis intervention during an event that might detract care and support from others during stressful times. In the end, such planning helps optimize the care for all.

\section{REFERENCES}

1. Hensrud D, Klein S. Extreme obesity: a new medical crisis in the United States. Mayo Clin Proc 2006;81(Suppl 10):S5-10.

2. Lurie N. H1N1 influenza, public health preparedness and health care reform. N Engl J Med 2009;361:843-5.

3. DeMaria E. Bariatric surgery for morbid obesity. N Engl J Med 2007;356: 2176-83.

4. McMahon M, Sarr M, Clark M, et al. Clinical management after bariatric surgery: value of a multidisciplinary approach. Mayo Clin Proc 2006;81(Suppl 10):S34-45.

5. Kahn CA, Oman JA, Rudkin SE, et al. Can ED staff accurately estimate the weight of adult patients? Am J Emerg Med 2007;25:307-12.

6. Block J, DeSalvo K, Fisher W. Are physicians equipped to address the obesity epidemic? Knowledge and attitudes of internal medicine residents. Prev Med 2003;36:669-75.

7. Berger E. Emergency departments shoulder challenges of providing care, providing dignity for the "super obese". Ann Emerg Med 2007;50(4):443-5.

8. Gougelet R. Modular medical systems (MEMS) for all types of catastrophic emergencies: a guide for community preparedness. Lebanon ( $\mathrm{NH})$ : Dartmouth Medical School, New England Center for Emergency Preparedness; 2009.

9. Gifford A, Gougelet R. Intensive care unit microcosm within disaster medical response. In: Geiling J, editor. Fundamental disaster medicine. Mount Prospect (IL): Society of Critical Care Medicine; 2009. p. 2.1-2.14.

10. Gifford A, Spiro P. Augmenting critical care capacity during a disaster. In: Geiling J, editor. Fundamental disaster medicine. Mount Prospect (IL): Society of Critical Care Medicine; 2009. p. 3.1-3.10.

11. Jbeili $C$, Penet $C$, Jabre $P$, et al. [Out-of-hospital management characteristics of severe obese patients]. Ann Fr Anesth Réanim 2007;26:921-6 [in French].

12. Nguyen $H$, Schweitzer M, Magnuson $T$, et al. Bariatric surgery: the needs of the obese patient. Critical Connections Aug 2007;1:12. Available at: http://www. sccm.org/criticalconnections. Accessed July 14, 2010.

13. Grant P, Newcombe M. Emergency management of the morbidly obese. Emerg Med Australas 2004;16:309-17.

14. Rubinson R, Hick J, Curtis R, et al. Definitive care for the critically ill during a disaster: medical resources for surge capacity: from a Task Force for Mass Critical Care summit meeting, January 26-27, 2007, Chicago, IL. Chest 2008; 133:32S-50S.

15. Erstad B. Dosing of medications in morbidly obese patients in the intensive care unit setting. Intensive Care Med 2004;30:18-32.

16. Snow V, Barry P, Fitterman N, et al. Pharmacologic and surgical management of obesity in primary care: a clinical practice guideline from the American College of Physicians. Ann Intern Med 2005;142:525-31. 
17. Devereaux A, Burns S, Gougelet R. Delivering acute care to chronically ill adults in shelters. In: Geiling J, editor. Fundamental disaster medicine. Mount Prospect (IL): Society of Critical Care Medicine; 2009. p. 11.1-11.14.

18. Devereaux A. Shelter medicine: beyond the first and second response. Chest Physician 2006;1:13.

19. Wolf L. The obese patient in the ED. Am J Nurs 2008;108:77-81.

20. Shaw L. Emergency preparedness \& evacuation issues of the bariatric specialty population. Presented at National Bariatric Nurses Association Conference. Orlando (FL), November 6, 2009.

21. Tsismenakis A, Christophi C, Burress J. The obesity epidemic and future emergency responders. Obesity 2009;17:1648-50.

22. Haber C. Bariatric transport challenges: part 1. EMS Mag 2008;37:67-71.

23. Chinook information. Available at: http://en.wikipedia.org/wiki/CH-47_Chinook. Accessed July 14, 2010.

24. Pieracci F, Barie P, Pomp A. Critical care of the bariatric patient. Crit Care Med 2006;34:1796-804.

25. Meroz Y, Gozal Y. Management of the obese trauma patient. Anesthesiol Clin 2007;25:91-8.

26. Brown C, Neville A, Rhee P, et al. The impact of obesity on the outcomes of 1153 critically ill blunt trauma patients. J Trauma 2005;59:1048-51.

27. Winfield R, Delano M, Dixon D, et al. Differences in outcome between obese and nonobese patients following severe blunt trauma are not consistent with an early inflammatory genomic response. Crit Care Med 2010;38(1):51-8.

28. Hogue C, Stearns J, Colantuoni E, et al. The impact of obesity on outcomes after critical illness: a meta analysis. Intensive Care Med 2009;35:1152-70.

29. Sifri Z, Kim H, Lavery R, et al. The impact of obesity on the outcome of emergency intubation in trauma patients. J Trauma 2008;65:396-400.

30. Dries D, Bracco D, Razek T, et al. Conventional explosions and blast injuries. In: Geiling J, editor. Fundamental disaster medicine. Mount Prospect (IL): Society of Critical Care Medicine; 2009. p. 7.1-7.26.

31. Gottschlich M, Mayes T, Khoury J, et al. Significance of obesity on nutritional, immunologic, hormonal, and clinical outcome in burns. J Am Diet Assoc 1993; 93:1261-8.

32. Azocar R, Shaffer D. Disasters produced by natural phenomena. In: Geiling J, editor. Fundamental disaster medicine. Mount Prospect (IL): Society of Critical Care Medicine; 2009. p. 9.1-9.15.

33. Geiling J, Nicolais V, Susla G. Critical care management of chemical exposures. In: Geiling J, editor. Fundamental disaster medicine. Mount Prospect (IL): Society of Critical Care Medicine; 2009. p. 4.1-4.18.

34. Beigel J, Sandrock C. Intentional and natural outbreaks of infectious disease. In: Geiling J, editor. Fundamental disaster medicine. Mount Prospect (IL): Society of Critical Care Medicine; 2009. p. 5.1-5.30.

35. Amundson D, Bracco D, Parrish J. Critical care management of radiological emergencies. In: Geiling J, editor. Fundamental disaster medicine. Mount Prospect (IL): Society of Critical Care Medicine; 2009. p. 6.1-6.18.

36. Onyike C, Crum R, Hochang B, et al. Is obesity associated with major depression? Results from the third National Health and Nutrition Examination Survey. Am J Epidemiol 2003;158:1139-47.

37. Owens D. Palliative care and mental health issues. In: Geiling J, editor. Fundamental disaster medicine. Mount Prospect (IL): Society of Critical Care Medicine; 2009. p. 12.1-12.18. 
38. Christian M, Farmer J, Young B. Disaster triage and allocation of scarce resources. In: Geiling J, editor. Fundamental disaster medicine. Mount Prospect (IL): Society of Critical Care Medicine; 2009. p. 13.1-13.18.

39. Devereaux A, Dichter J, Christian M, et al. Definitive care for the critically ill in a disaster: a framework for the allocation of scarce resources in mass critical care. Chest 2008;133:51S-66S. 